
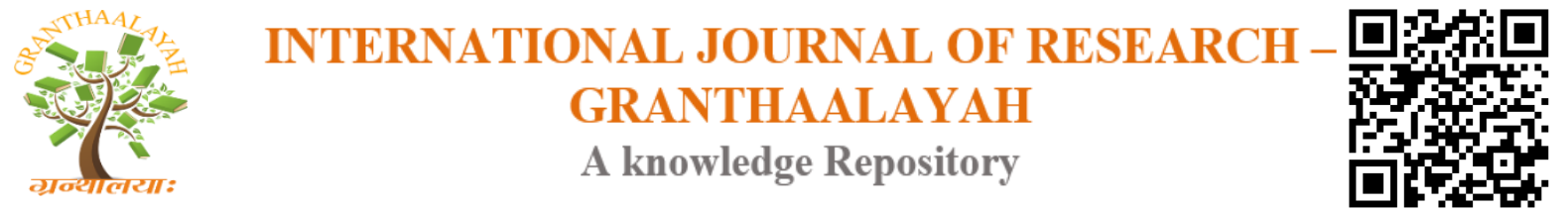

Science

\title{
COMPARISONS OF DYE PLUME MIGRATION AT THE NEAR-WALL REGION OF TWO DIFFERENT FLAT BEDS ROUGHENED WITH 7 MM AND 10 MM SYNTHETIC PLEXIGLAS BEADS
}

\author{
Okuroghoboye Diepreye Itugha ${ }^{1}$, Emmanuel Munakurogha Adigio ${ }^{2}$ \\ ${ }^{1}$ Faculty of Engineering, Civil \& Electrical/Electronics Engineering Department, Federal \\ University Otuoke, 400 University Boulevard, Otuoke, PMB 126, Bayelsa State, Nigeria \\ ${ }^{2}$ Faculty of Engineering, Mechanical/Marine Engineering Department, Niger Delta University, \\ Wilberforce Island, Bayelsa State, Nigeria.
}

\begin{abstract}
The detection of moving particles and the ability of processing the captured images using highspeed cameras can be an effective means of monitoring the sliding, and rolling movement of bedload sediment along river beds. In this study, two different types of synthetic Plexiglas Beads of size $7 \mathrm{~mm}$ and $10 \mathrm{~mm}$ with porosity 0.7125 and 0.8522 respectively were used for the evaluation of the velocity profiles in a flume bath. Potassium permanganate $(\mathrm{KMnO} 4)$ crystal of molecular weight 158.03 and density $1450 \mathrm{~kg} / \mathrm{m} 3$ was used as a passive dye-tracer. The cavity region of the flat plate was attached to the oscillatory mechanism in the flume bath where plume activities were monitored. When there is oscillatory flow, the crest of roughness elements induce elevation zones to force (advection) the flow upwards and pull it down at the trough phases as the tracer plume scales over the obstacles, establishing low and high pressure zones in the flow. The enhanced particles in the process mix and dissolve or decay faster with increasing oscillatory frequency, resulting into inter-particle collisions which initiate the conditions of advection and shear dispersion. This phenomenon could be a combination or coupling of the processes of gradient fluctuations enhanced through dispersion due to pressure in the macroscopic pore-fluid field caused by the oscillatory motion. The lightening as the dye-particles cascade in the porous medium is associated with mixing-gain due to the enhanced oscillatory motion. It was observed that the depth of the tracer-blob entrapment is inversely proportional to the friction velocity.
\end{abstract}

Keywords: Mass Transport; Plexiglas; Porous Flatbed; Near-Wall-Flow; Interstitial Hydraulics; Oscillatory Rig.

Cite This Article: Okuroghoboye Diepreye Itugha, and Emmanuel Munakurogha Adigio. (2019). "COMPARISONS OF DYE PLUME MIGRATION AT THE NEAR-WALL REGION OF TWO DIFFERENT FLAT BEDS ROUGHENED WITH 7 MM AND 10 MM SYNTHETIC PLEXIGLAS BEADS." International Journal of Research - Granthaalayah, 7(4), 147-161. https://doi.org/10.29121/granthaalayah.v7.i4.2019.884. 


\section{Introduction}

The motion of dye plumes as bed load particles is investigated under steady and spatially uniform turbulent flow into and above two flat porous beds with uniform Plexiglas balls of sizes $7 \mathrm{~mm}$ and $10 \mathrm{~mm}$. The experiments were designed to mimic and explore the sediment bed of the coastal zone which exhibits a large variation of sediment sizes. Migration of bed load particles and their respective classification over rough permeable layers on flat beds can be found in Nikora et al (2007b). Several research papers have interpreted the behavior of the cascading particles and transfer mechanisms as having fundamental importance in river morphodynamics, such as resulting from variations of bed materials, particles rolling, and migrating low jump successions (saltations) along the bed of open channels. For instance, Keramaris $(2015,2017)$ compared flow experiments in impermeable and permeable beds and found significant changes in the velocity distribution and turbulent characteristics due to porosity differences. Nielson et al (2001) used sand-roughened beds to show the effects of infiltration while studying sediment migration. There is also the contentious claim that hydraulic roughness depends strongly on the velocity profile of the logarithmic region than on bed grain size. However, turbulence measurements of some rivers by Smart (1999) supported those of Nezu and Nakagawa (1993) which show that turbulence intensity is highest near the channel bed but decreases on approaching the water surface. Choi and Waller (1997) observed that turbulence intensity into the depth of porous medium is dependent on the Darcy number of the elements and not related to the Reynolds number (speed) and shape of the velocity profile in the region. Prinos et al (2003) also confirmed that the fluid's kinetic energy penetrating the porous medium increases with the Darcy number which is dependent on the structural geometry of the roughness elements (the pore size of the cavities or gaps between the elements).

Svenson and Rahm (1991) used the benthic sub-layer to study water quality and mass transfer problems due to dispersion mechanisms over thin vertical interfacial layers. Shams et al (2003) investigated the near-edge flow domain of a porous medium by introducing shear-flow across arrays of rods to obtain the flow pattern within, using a PIV. Their work affirms that a secondary motion arises during the shear flow in the square array of rods which is capable of heat and mass transfer between the first and second rows except contaminant is trapped during the flow. Infiltration, according to some researchers (Nielson et al., 2001, Suga and Kuwata, 2014; Suga, 2016), has no main effect on the migration direction of bed-sand except to reduce their effective transport.

Stansby (2003) observed that in a turbulence based two layer mixing-length model, the horizontal diffusion scale (length scale) is a multiple of the vertical mixing length scale, implying that horizontal and vertical mixings due to applied pressure gradients are responsible for the eddy viscosity. A study involving oscillatory-grid aided turbulence by An and Chen (2004) has also shown the phenomenon of turbulence-driven diffusion. Some researchers (Davis and James, 2003; Shim and Duan, 2017) investigated the role of the interior rows of the porous medium in dissipating energy where it was shown that the slip velocity is dependent on the hydrodynamic resistance of the surface elements and not the inner layers. Pechlivanidis et al. (2012) reported from an open-channel flow experiments using Particle Image Velocimetry, that the velocity is a function of the roughness height and the total flow depth. 
This study relates to the motion of the fluctuating parcels of dye tracer just above the roughness boundary layer that has been likened to a logarithmic scale, in the interfacial and subsurface sublayers.

\section{Experimental Set-Up with Procedure}

Potassium permanganate $\left(\mathrm{KMnO}_{4}\right)$ crystals of molecular weight 158.03 , and density $1450 \mathrm{~kg} / \mathrm{m}^{3}$ was used a passive dye-tracer. Some $0.05 \mathrm{~g}$ of dry $\mathrm{KMnO}_{4}$ was scooped using a spatula to a flat 9 $\mathrm{mm}^{2}$ shape of cotton-wool and wrapped into a small ball with a finger tube-grip material (ball diameter $<5 \mathrm{~mm}$ ). The ball is trapped into the porous cavity on a flat plate and the trap position was varied from the top to the near bed as the oscillatory speed is regulated.

Tap water is introduced into the tank and allowed to stabilize and subsequently varied from $20.0 \mathrm{~cm}$ to $40.0 \mathrm{~cm}$. The crystal particles in the tube-grip dissolve and settle onto the bed being denser, prior to inception of the oscillatory movement. The air pressure valve is released, and the speed regulator is set and varied from $0.5 \mathrm{~m} / \mathrm{s}$ start-mark. The oscillatory rig responds at $0.22 \mathrm{~m}$ stroke length. The two black stripe marks on the external wall of the flat plate (which holds the porous elements) enable the determination of the center of oscillation.

At the $3 \times 7 \mathrm{~mm}$ plexi-ball stacks, the tracer blob position within the cavity region was variably trapped at distances $0.021 \mathrm{~m}, 0.014 \mathrm{~m}, 0.007 \mathrm{~m}$ away from the impermeable bed and $0.000 \mathrm{~m}$ (i.e., at the bottom). For the $2 \times 10 \mathrm{~mm}$ stacks, the position was varied by $0.02 \mathrm{~m}, 0.01 \mathrm{~m}$ and at flume bed $(0.000 \mathrm{~m})$, such that the earlier arrangement is $0.001 \mathrm{~m}$ higher. The porosity of the $7 \mathrm{~mm}$ arrangement estimates to 0.7125 and that of $10 \mathrm{~mm} 0.8522$. The Measurements were taken vertically away from the roughness bed region, aided by millimeter-squared graphic transparencies. The flow distances were measured across the longitude of flume cross-section within the cavity area. The velocity record obtained was varied at every oscillatory time interval of $0.5 \mathrm{~m} / \mathrm{s}$ up to $4.0 \mathrm{~m} / \mathrm{s}$. The oscillatory events were also monitored with a stop-clock and recorded. The still water level in the tank was stabilized at a maximum height of $0.40 \mathrm{~m}$.

The Plexiglass frame of the tank opposite the test-window was curtained and the overhead light source dimmed to reduce lighting source that may have direct effect on the CCDC lens by reflection, to account for more contrast of the glass beads. The tube-grip $\mathrm{KMnO}_{4}$ ball was installed initially at the top of the cavity formed with the glass beads and then relocated further vertically down the bed at the completion of each group of experiments. A total of about seventy (70) averaged results were analyzed.

The distribution of the plume particles due the velocity describes the boundary layer thickness. The motion of the plumes tending towards the vertical direction may be considered more rapid compared to those lateral along the bed.

\section{Parameter evaluation and scaling of CCDC plume events}

The wavy plume parcels visualized in the free-fluid phase above the roughness elements in the mean direction of oscillatory flow were assumed to represent floating particles of the passive test substance. These images were transferred from the VCR analogue tapes into a Sony DV Camcorder DCR-PC55E and later digitized by copying into computer using i•LINK (IEEE1394) 
cable connector. The images of the plume parcels were then digitally enhanced and tracked from pre-, and through post-flow initiation stages. The Arc Soft Showbiz 2 software, a professional video image sequencing application, was used to digitally process the CCDC video clips. The motions of the plume parcels were tracked along the vertical flow fields and measured just above the water-porous elements interface aided by millimetre markers on the Plexiglass test-window pane relative to their respective trap-posts. The visual area of interest here is the plume parcel displacement from the trap-posts to and above the interfacial layer. Plume parcels were therefore followed from the trap-posts per complete cycle of oscillation and with increasing flow rates.

\section{Mathematical Analysis}

The flume medium has the fluid phase and the porous material-in-fluid region, where $h_{1}=$ height of the water only column, $\mathrm{h}_{2}=$ porous preform (roughness)-in-fluid region medium. The governing equation related to the influx in the roughness medium at steady state may take the Darcy velocity form:

$$
\mathbf{q}=-\mathbf{K}(\psi) \nabla(\psi+z)
$$

Where $\mathbf{q}=-\mathbf{K} \nabla h$ for $\mathrm{x}-\mathrm{z}$ heterogeneous plane of the porous elements, $\psi=p / g \rho=$ pressure head, $(h=z)$ elevation head. Assuming isotropy, $\mathbf{K}$ (hydraulic conductivity) is defined as a function of the pressure head $\mathbf{K}(\psi)$. Such that the boundary conditions in this case relates to a well distributed instantaneous tracer source bounded by

$$
\frac{\partial c_{1}}{\partial z}=0 @ z=h_{1}+h_{2} \text { and } \frac{\partial c_{2}}{\partial z}=0 @ z=0
$$

The flume medium has the fluid phase and the porous material-in-fluid region. The steady and progressive transfer of mass at the porous-fluid interface may be described by the condition

$$
\varepsilon_{v}=\frac{\partial c}{\partial z_{v}} @ z \leq \pm h_{2}
$$

For the initial conditions the mass concentration fields in relation to both regions may be described by their respective compressibility constants as

$$
c_{1}(x, z, t=0)=\alpha \frac{m_{1}}{h_{1}} \delta \text { and } c_{2}(x, z, t=0)=\beta \frac{m_{2}}{h_{2}} \delta
$$

The compressibility constants are assumed equal if tracer mass are found in both mediums (i.e., $\alpha=\beta=1$ ), but 0 or 1 respectively depending on the region tracer mass is present. 
The velocity describing the mass distribution in first region may be related to the form:

$u_{1}(z)=k_{s} u_{*}+\frac{u_{*}}{0.077}\left(\frac{z-h_{2}}{h_{1}}-0.5\left[\frac{z-h_{2}}{h_{1}}\right]^{2}\right)$

The assumed uniform flow profile from the interface to the porous medium takes the form (as in Engelund, 1970):

$u_{2}(z)=\bar{u}+u_{0} e^{k\left(z-h_{2}\right)}$

\section{Fitted Flow Parameters}

The cavity region is the visual monitoring area of interest, such that the displacement of the plume parcels along the vertical away from the TP was tracked with time using Arcsoft Showbiz 2. For porosity determination, the flat plate used was partitioned into two panels. Total volume of plate is $1320 \mathrm{~m}^{3}$, and for the $7 \mathrm{~mm}$ arrangement as example, the width $=98 \mathrm{~mm}$, length $=59 \mathrm{~mm}$, depth $=21 \mathrm{~mm}$. So the volume occupied by the Plexiglass balls (pore volume) divided by total volume estimates the porosity to 0.7130 .

The measured variables were the variable vertical plume heights, $h_{p l m}$, the maximum velocity, $\mathrm{u}_{\mathrm{m}}$ applied, and time, $(\mathrm{T}=2 \mathrm{t})$ records. At least five (5) tests were performed on each case and measurements averaged.

\section{Analysis and Discussions}

\section{Comparisons of the Test Cases at the $7 \mathrm{~mm}$ and $10 \mathrm{~mm}$ walls}

The estimated porosity for the $7 \mathrm{~mm}$ category is 0.7125 and that of $10 \mathrm{~mm}$ category is 0.8522 . Estimates from the governing parameters from the experiments using CCDC shows that as the position of the tracer blob is shifted through the porous preforms from the top towards the bottom the friction velocity increases with the oscillatory velocity.

\section{Tracer blob trapped at $0.021 \mathrm{~m}$ above bed level of the $7 \mathrm{~mm}$ arrangement}

The averaged relative heights of the plume, $h_{p l m} / H_{w}$ (dimensionless) varied from 0.013 to 0.025 during the period of oscillation, as the velocity was regulated from $4 \mathrm{~m} / \mathrm{s}$ to $0.3 \mathrm{~m} / \mathrm{s}$ respectively. The curve in Figure 1a illustrates that the flow near the bed may be controlled by quasiequilibrium in turbulent kinetic energy (TKE) which balances the dissipation in the rough cavity. This implies that TKE rises to a maximum as the velocity becomes higher being sustained by diffusion. This behavior increases the length scale with distance away from the near-wall region. The averaged maximum velocity within the confidence interval estimates to $2.033 \pm 0.439 \mathrm{~m} / \mathrm{s}$. The fitted parameters in this category include $\kappa=0.41, \frac{k_{s}}{30}=0.2613, u_{*}=0.0101 \mathrm{~m} / \mathrm{s}$ and roughness height, $\bar{h}_{R_{p l m}}=\bar{z}=0.0177(\mathrm{~m})$. 


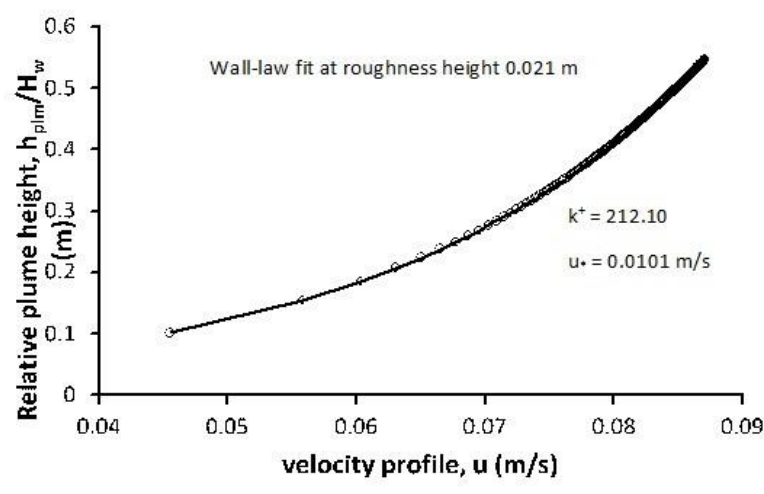

(a)

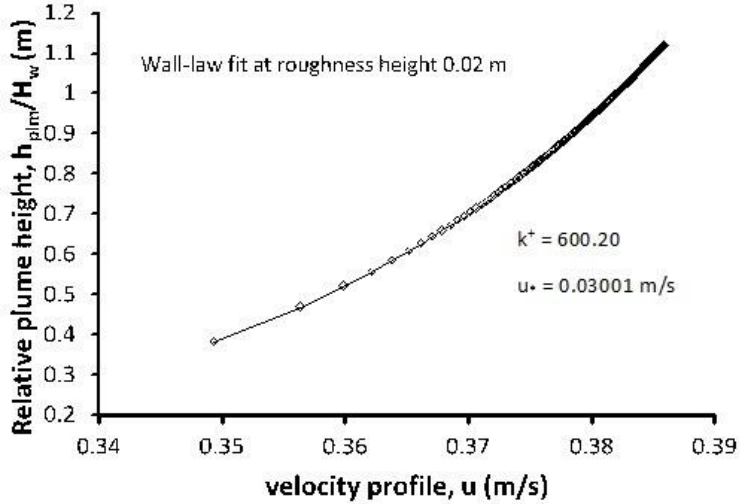

(b)

Figure 1: Velocity Profile of Plume from Tracer Blob Trapped At $0.021 \mathrm{~m}$ and $0.02 \mathrm{~m}$ of the $7 \mathrm{~mm}$ and 10mm Stacks Respectively Above Bed

The estimated amplitude Reynolds number in this case was $2.367 \times 10^{3}$ with a mean value within the confidence interval estimate (WCIE) as 3961.1 \pm 652.30 . The Froude, number, Fr, is $5.7783 \mathrm{x}$ $10^{-3}$ with a mean varying WCIE as $0.0024 \pm 0.0008$, obtained from the experiments. The mean velocity input also from experimentation was $0.076 \mathrm{~m} / \mathrm{s}$. The ratio of the roughness height to the friction length, $k^{+}$is shown in Figure 1a, indicating fully rough flow, with Darcy permeability WCIE as $0.00023 \pm 2.5 \times 10^{-5} \mathrm{~m} / \mathrm{s}$.

The profile of the curve shows the influence of the effect of rough bed material region as it moves away towards the outer regions. At the bed region the curve seem to deviate from the logarithmic behaviour. A repeat of this characteristic behaviour is also likely as the plume approaches reasonable heights above the roughness elements unto the near surface regions. Figure 1a also shows this influence of the rough elements as the time averaged velocity profiles increase with distance along the vertical cross-section of the rough cavity domain, that is, away from the surface of the rough elements. The reality of this situation is seen from the fitted curve as it intersects the plume axis at some finite value $\left(h_{p l m} / H_{w}=0.26\right)$ close to the roughness surface where the velocity profile loses its direct proportionality character with the vertical plume-axis. The reduced magnitude of velocity profiles at the near-surface region is an indication of turbulent shear stress penetration affecting the porous medium which becomes weaker as Darcy permeability also decreases $\left(3.175 \times 10^{-4}\right.$ to $\left.9.75 \times 10^{-5}\right)$.

The reduction in the Darcy accounts that plume permeability was affected by the oscillatory velocity profile. At the porous interface the flow is generally assumed to show some semblance of mixing layer type than that of boundary layer, according to Prinos et al (2003).

At some point along the roughness surface the velocity profile shifts away from the interfacial curve to a position where momentum or the velocity deviates from the logarithmic law (Raupach et al 1991, Prinos et al, 2003). This shifting in the mean position of the velocity relative to the vertical axis has been referred to as the beginning of the logarithmic layer which portrays the dampening effects of the roughness elements on the horizontal oscillatory shear flow (Prinos et al, 2003). 
Figure 2a portrays a non-dimensional version of the near-bed flow structure described in Equation (3). The mean velocity was normalized with the friction velocity and plotted against the normalized plume heights. The logarithmic law fit compares favorably with the mean velocity plot in Figure 1a both of which has $\mathrm{R}^{2}$ values of 0.98 and 0.91 respectively. The curve describes how turbulence evolves with the mean oscillatory velocities near the bed and continues across the developing boundary layer from the roughness surface. At some critical point near to flow reversal, the energetic/turbulent plume in the free stream approach a uniformly distributed flow stream. This observation has also been accounted by Jensen et al (1989) and attributed to an inner-flow characteristic behaviour.

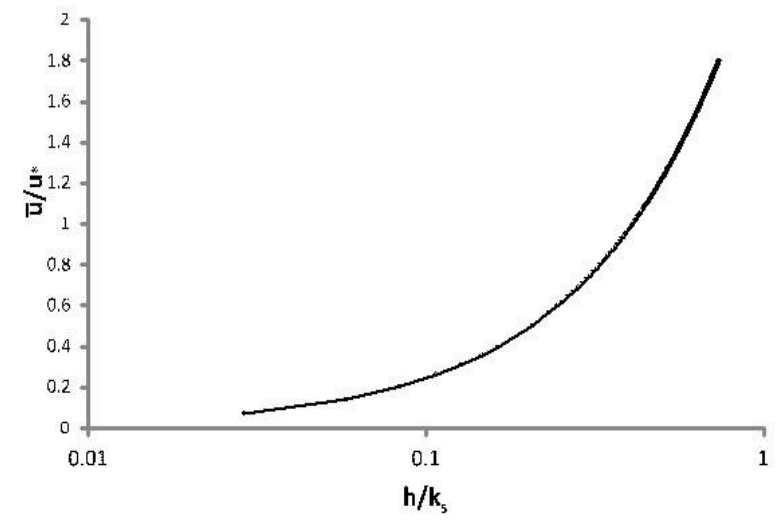

(a)

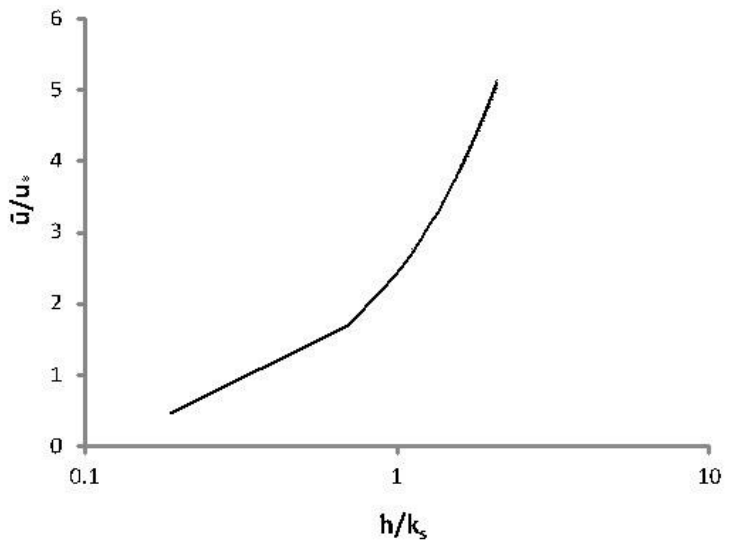

(b)

Figure 5: Semi-log Plot of None Dimensional Velocity Profile in Wall Units At $0.021 \mathrm{~m}$ and $0.02 \mathrm{~m}$ of the $7 \mathrm{~mm}$ and $10 \mathrm{~mm}$ Stacks Respectively Above Bed

\section{Tracer blob at trough position $0.02 \mathrm{~m}$ of the $10 \mathrm{~mm}$ bed}

Plume heights $h_{p l m} / H_{w}$ in this arrangement varied between the ranges 0.018 to 0.038 . The maximum mean stream oscillatory velocity here was also regulated from $4.0 \mathrm{~m} / \mathrm{s}$ to $0.3 \mathrm{~m} / \mathrm{s}$ accordingly. A clear decrease in the velocity profiles can be identified as the characteristic curve deviate from linearity towards the near-wall region Figure $\mathbf{1 b}$.

The shift in the mean position of the velocity relative to the vertical axis shows the onset of dampening effects of the roughness elements on the horizontal oscillatory shear flow. The $\kappa=$ $0.41, \frac{k_{s}}{30}=0.3519, \mathrm{u}_{*}=0.03001 \mathrm{~m} / \mathrm{s}$ and $\bar{z}=0.0277$ were fitted to the log-law.

The Froude number increased WCIE to $0.0012 \pm 0.0004$ with the amplitude Reynolds number varying by $6056.8 \pm 918.2$. In comparison to the case with the $0.021 \mathrm{~m}$ roughness height of $0.007 \mathrm{~m}$ diameter spheres, the Reynolds and Froude numbers were seen to increase, which may be due to pore-size differences. The mean velocity from the distance-time graph was $0.134 \mathrm{~m} / \mathrm{s}$.

We consider Figure $\mathbf{2 b}$ in comparison to Figure $\mathbf{1 b}$ which shows the shapes of the curves to be in good agreement with each other. Figure $\mathbf{2 b}$ portrays a non-dimensional version of the near-bed flow structure in a semi-log plot. The mean velocity was normalized with the friction velocity and plotted against the records of normalized plume heights. The viscous sub-layer and the developing 
constant stress region are clearly seen in the limit of increasing velocity record in the free stream.

In Figure $2 \mathbf{b}$, the $\frac{\bar{u}}{u_{*}}$ is an order of magnitude greater than the mean velocity record plot in Figure

1b. The curve describes how turbulence evolves with the mean oscillatory velocities near the bed and continues across the developing boundary layer from the roughness surface. At some critical point near to flow reversal, the energetic/turbulent plume in the free stream approach a uniformly distributed flow stream.

\section{Tracer blob trapped at trough position $0.014 \mathrm{~m}$ of the $7 \mathrm{~mm}$ bed}

The velocity profiles show identical characteristic curve shapes compared with the former. The fluctuating velocity profiles of the plume parcels tracked relative to the vertical axis due to the oscillatory plate is shown in Figure 3a. The variation of plume heights, $h_{p l m} / H_{w}$ here was from 0.021 to 0.038 as the maximum mean stream oscillatory velocity was regulated from $4 \mathrm{~m} / \mathrm{s}$ to 0.3 $\mathrm{m} / \mathrm{s}$ respectively. The fitted parameters in this category include $\kappa=0.41, \frac{k_{s}}{30}=0.0752, \mathrm{u}_{*}=0.0092$ $\mathrm{m} / \mathrm{s}$ and $\bar{z}=0.0304$. The amplitude Reynolds number WCIE is $7144.33 \pm 1266.81$ while the Froude number is $0.0008 \pm 0.0003$. The mean velocity fit was $0.085 \mathrm{~m} / \mathrm{s}$. The shear stress, $\tau$ decreased (0.800 to 0.058 ) as with Darcy permeability $0.0004 \pm 4.8^{*} 10^{-5} \mathrm{~m} / \mathrm{s}$ as the flow into the porous medium weakens. The present case of $k^{+}$(Figure 3a), also indicates fully rough flow but the roughness parameter $k^{+}$slumped to show the effect of the difference in the roughness height in comparison to the case in Figure 1a. In comparison with Figure 2a, the graph shown in Figure 4a seems to demonstrate late appearance of the developing logarithmic boundary layer which subsequently describes the approach to flow reversal following the uniformly distributed plume in the fluid stream. The increase in the velocity below the interface is described by the Reynolds value and may be attributed to the developing constant stress across the free-stream.

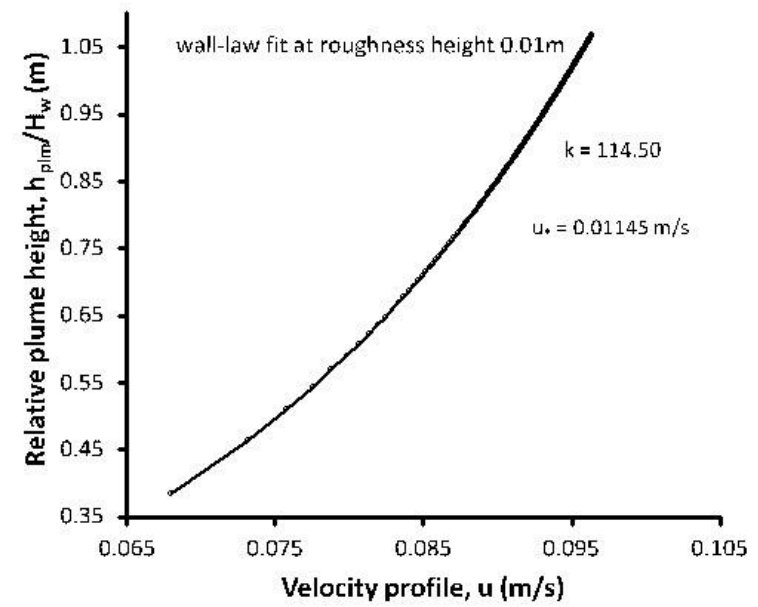

(a)

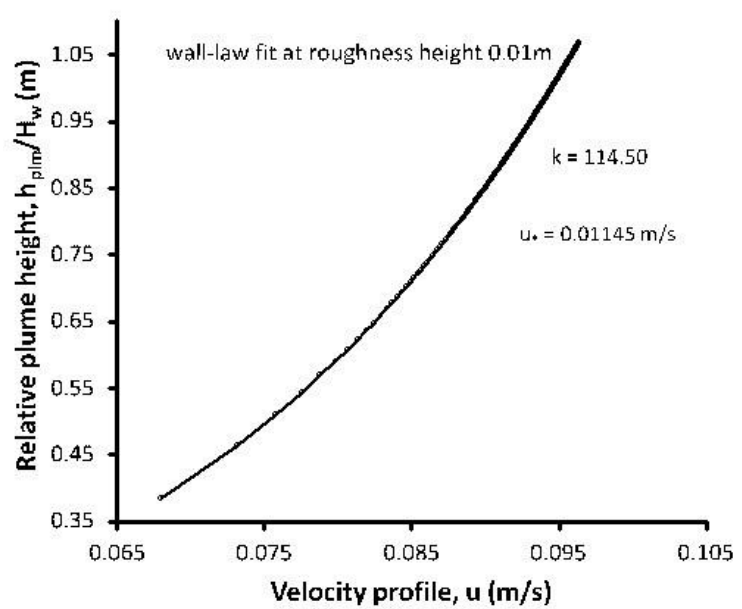

(b)

Figure 3: Velocity profile of plume trapped at $0.014 \mathrm{~m}$ and $0.01 \mathrm{~m}$ of the $7 \mathrm{~mm}$ and $10 \mathrm{~mm}$ stacks respectively above bed 


\section{Tracer blob at trough position $0.01 \mathrm{~m}$ of the $10 \mathrm{~mm}$ bed}

The profile of the velocity was identical to the previous cases where the characteristic curve deviates from linearity on approach to the near-wall region (Figure 6b). The shift in the mean position of the velocity relative to the vertical axis shows the dampening effects of the roughness elements on the horizontal oscillatory shear flow. The depth to source is accounted such that the depth averaged plume heights $h_{p l m} / H_{w}$ range from $0.038-0.058$ with the maximum mean stream oscillatory velocity regulated between $4 \mathrm{~m} / \mathrm{s}$ to $0.3 \mathrm{~m} / \mathrm{s}$ respectively.

The parameters imputed in this case relate to $\kappa=0.41, \frac{k_{s}}{30}=0.0338, \mathrm{u}_{*}=0.0115 \mathrm{~m} / \mathrm{s}$ and $\bar{z}=0.0499$. The The Froude number WCIE increased to $0.00026 \pm 8.3 \mathrm{E}-5$ as the position of plume crest moved further away from the surface elements with the amplitude Reynolds number varying between the confidence interval limits of $11906.6 \pm 2139.4$. The mean flow rate evaluated from the plotted instance in this case was $0.114 \mathrm{~m} / \mathrm{s}$ respectively. In comparison to Figure $\mathbf{4 b}$, Figure $\mathbf{3 b}$ seems to show the viscous sub-layer with the developing logarithmic layer as mean velocity increases towards the free stream. In Figure $\mathbf{4 b}$ however, the logarithmic boundary layer seems to develop at the later stages as the flow approach reversal with the uniformly distributed plume in the fluid stream.

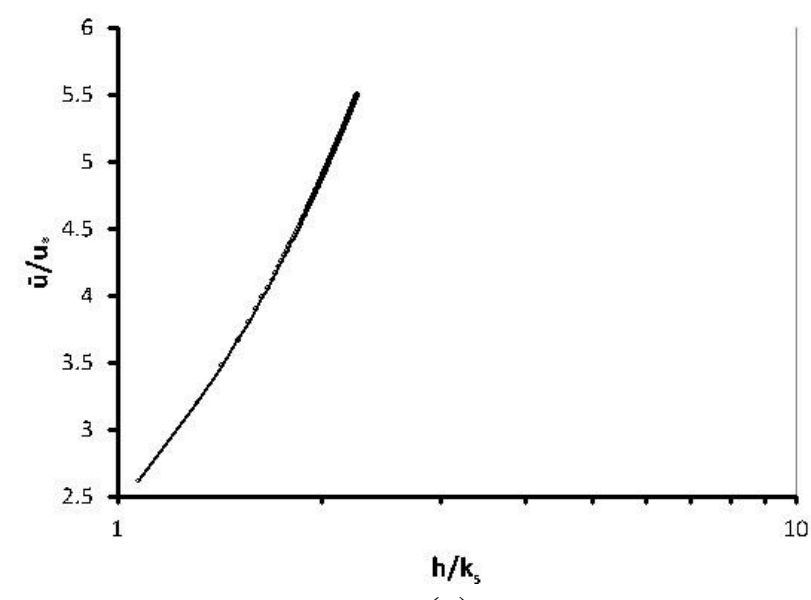

(a)

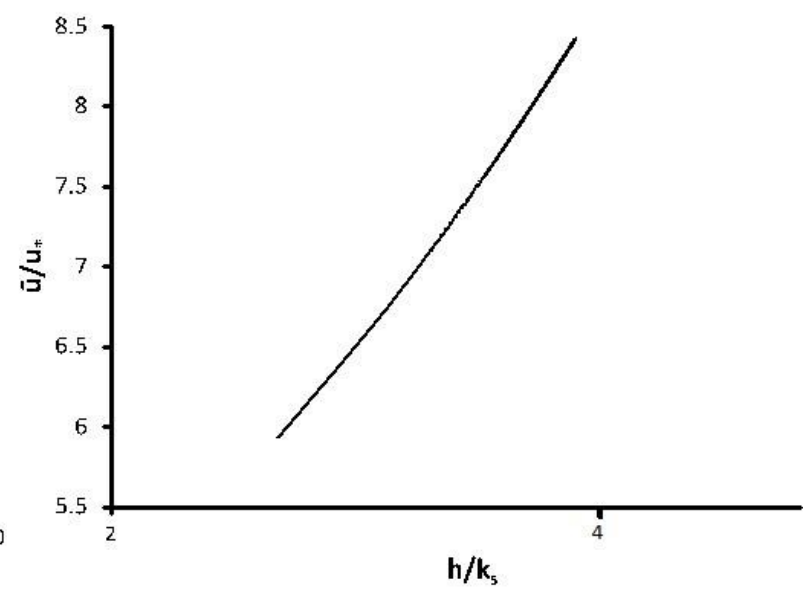

(b)

Figure 4: None Dimensional Velocity Profile in Wall Units at $0.014 \mathrm{~m}$ and $0.01 \mathrm{~m}$ above Bed in Semi-log Plot

\section{Tracer blob trapped at trough position $0.007 \mathrm{~m}$ of the $7 \mathrm{~mm}$ bed}

A clear decrease in the velocity profiles can be identical as the characteristic curve deviate from linearity from the mainstream flow to the near-wall region (Figure 5). The shift in the mean position of the velocity relative to the vertical axis shows the dampening effects of the roughness elements on the horizontal oscillatory shear flow. The depth to source is accounted such that the depth averaged plume heights $h_{p l m} / H_{w}$ range from 0.039 to 0.053 as the maximum mean stream oscillatory velocity was regulated from $4 \mathrm{~m} / \mathrm{s}$ to $0.3 \mathrm{~m} / \mathrm{s}$ respectively. The parameters imputed in this case relate to $\kappa=0.41, \frac{k_{s}}{30}=0.0235, \mathrm{u}_{*}=0.0082 \mathrm{~m} / \mathrm{s}$ and $\bar{z}=0.0436$. 


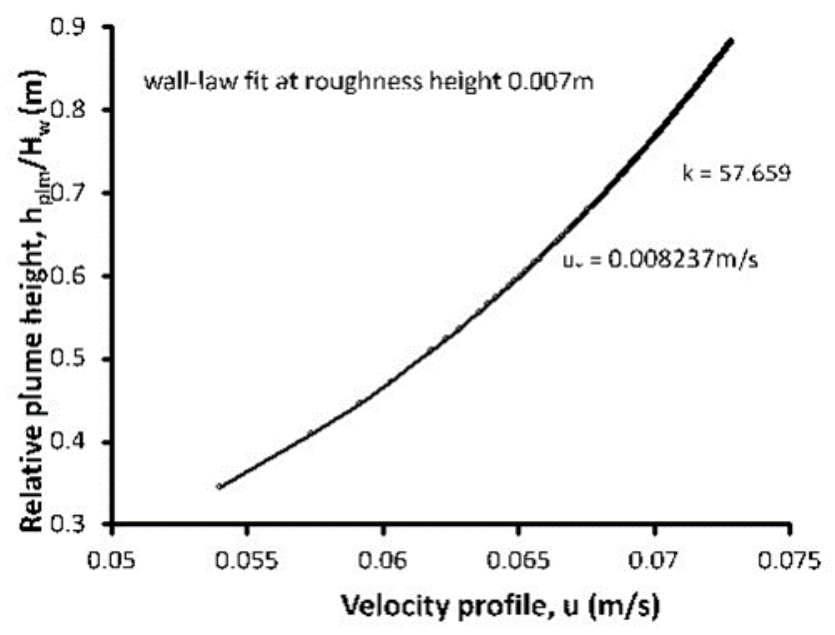

Figure 5: Velocity Profile of Plume Trapped at Roughness Height $0.007 \mathrm{~m}$ above Wall in $7 \mathrm{~mm}$ Arrangement

The amplitude Reynolds number WCIE is $10612 \pm 2073.3$ and the Froude number, $0.0003 \pm 8.2^{*} 10^{-}$ 5 . A mean velocity fit of $0.096 \mathrm{~m} / \mathrm{s}$ was inputted. The plot of the wall units show the development of the constant stress boundary layer, as in Figure 6, that compares reasonably well with the mean velocity profile plot in Figure 8. The behaviour of the plume in this case may be due to the position of the blob further away from the interface where the velocity within the porous spheres increases.

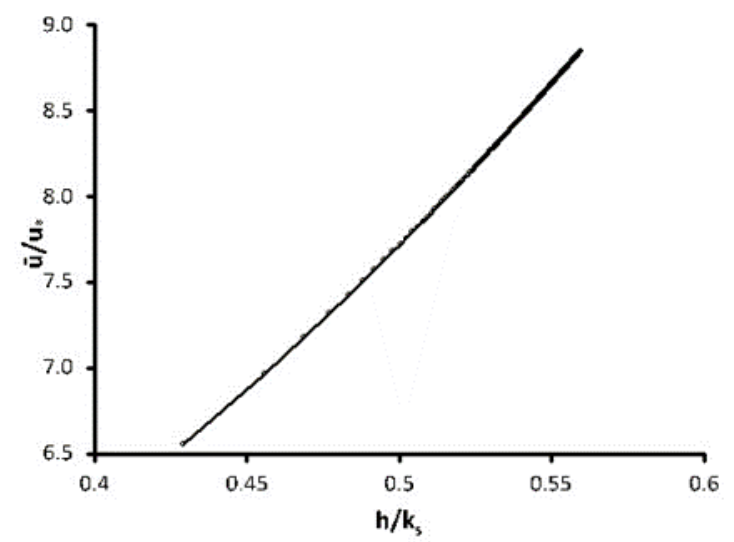

Figure 6: None dimensional velocity profile in wall units at $0.007 \mathrm{~m}$ above bed in semi-log plot

\section{Tracer blob trapped at bed level (zero height) of $7 \mathrm{~mm}$ preform}

The relative plume heights $h_{p l m} / H_{w}$ varied between the ranges 0.04 to 0.06 with the maximum mean stream oscillatory velocity regulated from $4 \mathrm{~m} / \mathrm{s}$ to $0.3 \mathrm{~m} / \mathrm{s}$ respectively. A clear decrease in the velocity profiles can be identified as the characteristic curve deviate from linearity in the mainstream flow to the near-wall region (Figure 7a). The shift in the mean position of the velocity relative to the vertical axis shows the dampening effects of the roughness elements on the horizontal oscillatory shear flow. The $\kappa=0.41, \frac{k_{s}}{30}=0.0482, \mathrm{u}_{*}=0.0139 \mathrm{~m} / \mathrm{s}$ and $\bar{z}=0.0508$ were fitted to the log-law. The amplitude Reynolds and the Froude numbers WCIE are 12094 \pm 2200.6 and $0.00025 \pm 7.5^{*} 10^{-5}$ respectively. The profile in Figure 8a also depicts the increase in the 
velocity at close to the permeable region, almost a roughness height away from the roughness surface. The constant stress layer compares well with the previous cases.

Figure 9a describes the normalized velocity distribution and plume height in wall units for the positions $0.021,0.014$ and $0.007 \mathrm{~m}$ of the entrapment tracer blob. The curves show that the velocity distribution curve approach constant stress at increasing wall-coordinate heights. This observation agrees comfortably with Fredsoe (1984), Jensen et al (1989) and those of Prinos et al (2003) who also described these relations in rough wall conditions. The lines on the curves are associated to the development of the constant stress describing the onset of the logarithmic layer across the free stream.

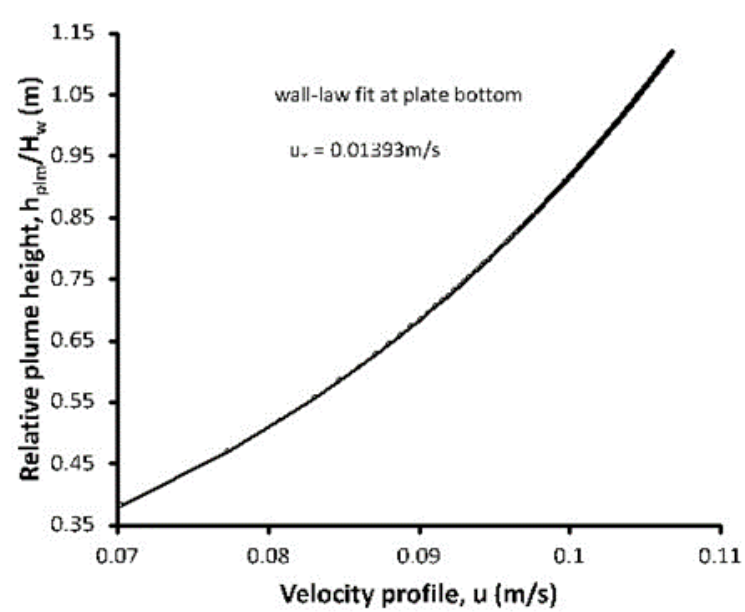

(a)

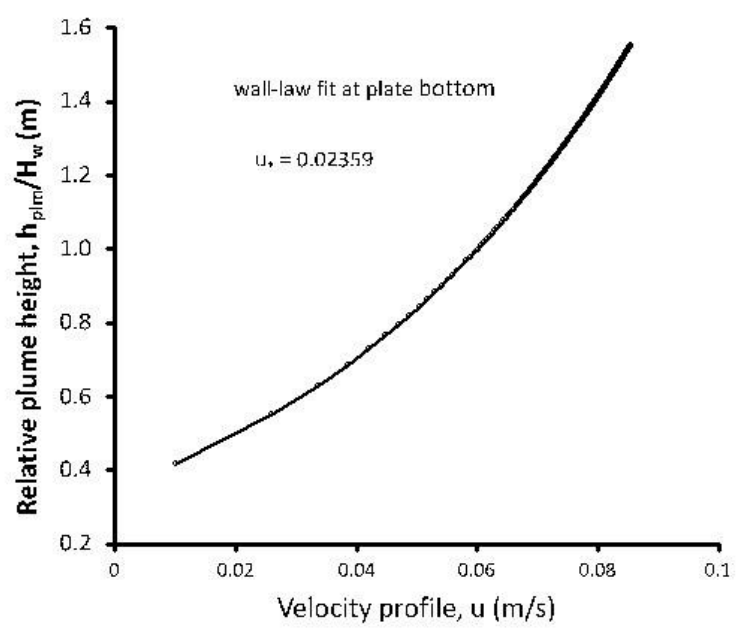

(b)

Figure 7: Velocity profile of plume trapped at flume base $(0.00 \mathrm{~m})$ of the $7 \mathrm{~mm}$ and $10 \mathrm{~mm}$ roughened plate respectively

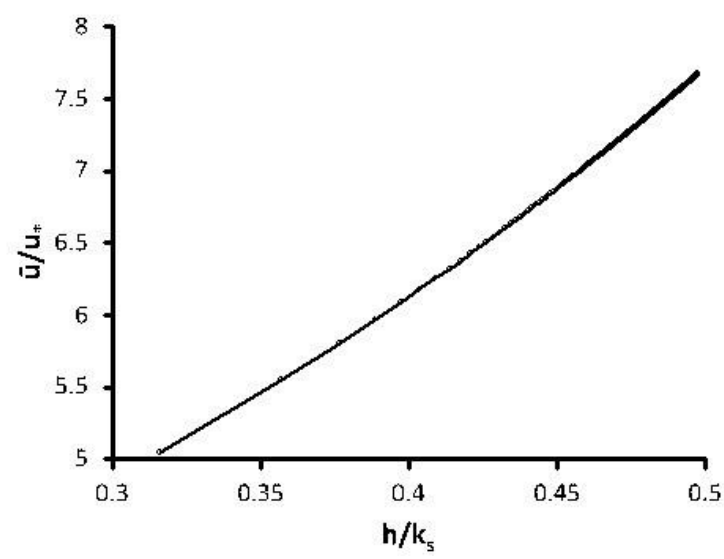

(a)

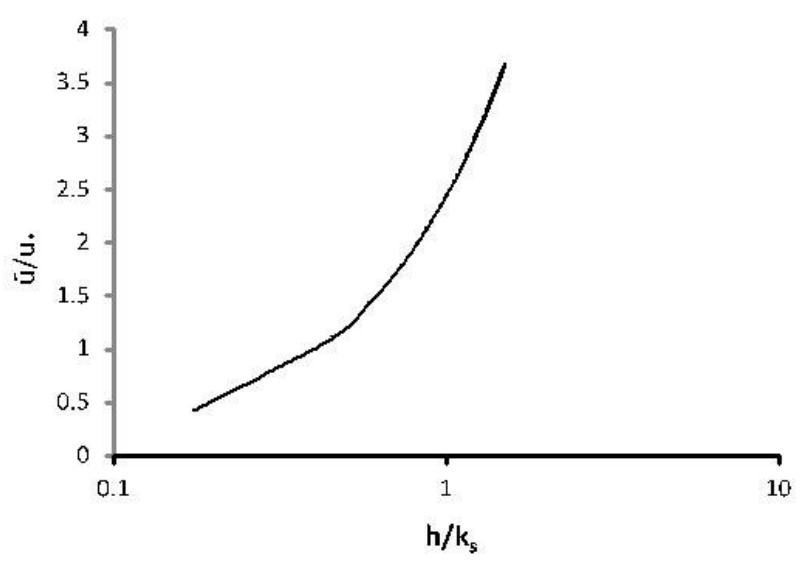

(b)

Figure 8: None Dimensional Velocity Profile in Wall Units at The Bottom of the $7 \mathrm{~mm}$ And10 mm Cases Respectively.

The friction factor at the roughness bed region was also plotted against the ratio of the particle amplitude in the oscillatory flow and the roughness size as shown in Figure 10a. The figure describes the behaviour of the friction factor at the four positions of the $0.007 \mathrm{~m}$ bed element 
arrangement. The curves show that the friction factor reduces as the amplitude factor increases. The sudden drop in the friction factor at about $\frac{a}{k_{s}}=108$ as traced by the dotted curve appears in all the curves and indicates the transition from the laminar boundary layer into turbulent boundary. A similar phenomenon occurred in the region of the boundary layer thickness at Reynolds numbers above $5.0 \times 10^{3}$ as seen in Figure 11a when the development of boundary layer depth, $\delta$, started into the free stream.

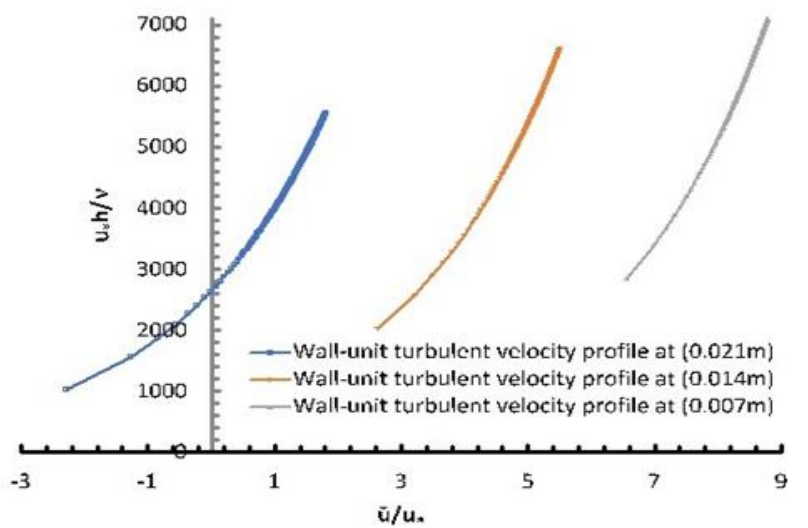

(a)

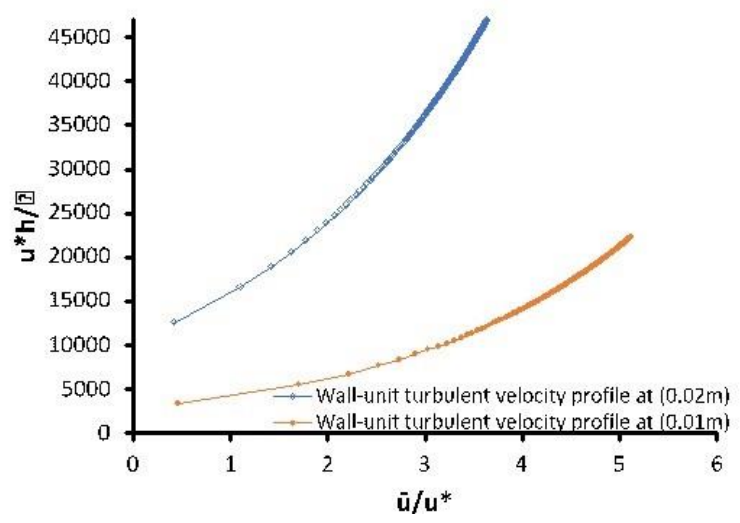

(b)

Figure 9: Comparisons of Velocity Profile in Wall Coordinates at Varying Depths for the 7mm and 10mm Cases Respectively

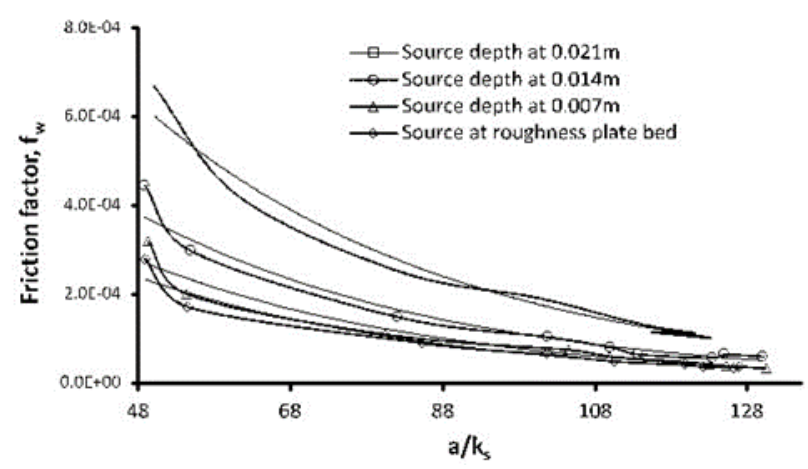

(a)

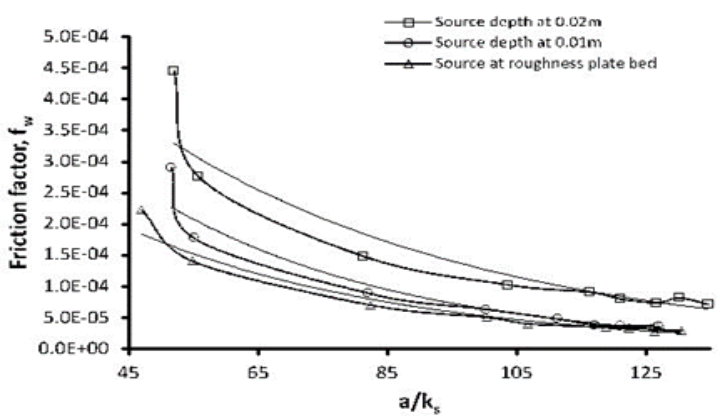

(b)

Figure 10: Friction Factor Relation with $\mathrm{A} / \mathrm{K}_{\mathrm{s}}$ At Various Tracer Source Depths for the $7 \mathrm{~mm}$ and 10mm Porous Preform Respectively

\section{Tracer blob at bed level (zero height) of the $10 \mathrm{~mm}$ bed}

The heights of the plume regime relative to the water depth here varied between 0.046 to 0.075 in response to the regulated oscillatory velocity $(4 \mathrm{~m} / \mathrm{s}$ to $0.3 \mathrm{~m} / \mathrm{s})$ respectively. Figure $7 \mathbf{b}$ illustrates the oscillatory flow stream as depicted by the tracer plume waves across the rough cavity flume domain. The fitted parameters to the analytical log law in this category include $\kappa=0.41, \frac{k_{s}}{30}=$ $0.1159, u_{*}=0.0236 \mathrm{~m} / \mathrm{s}$ and $\bar{h}_{R_{p l m}}=\bar{z}=0.0609$. 
The profiles in Figures $\mathbf{8 b}$ depict reasonably corresponding distribution of the velocity profiles. The early appearance of the viscous sub-layer from the surface of the roughness elements and the developing constant stress region are clearly represented as described by the curves. In both cases the curves describe how turbulence evolves with the mean oscillatory velocities near the bed and continues across the developing boundary layer from the roughness surface. At some point near to flow reversal however, the plumes in the free stream approach a uniformly distributed flow stream. Figure $7 \mathbf{b}$ describes the normalized velocity distribution and plume height in wall units for the positions 0.02 and $0.01 \mathrm{~m}$ of the entrapment tracer blob. The curves show that the velocity distribution curves approach constant stress at increasing wall-coordinate heights. The heights in wall unit are about an order of magnitude more than the distributions described in Figure 0a. This development may be attributable to the porosity differences due to roughness material sizes of the respective beds. The paths of the curves narrate the history of the developing logarithmic regions above the viscous sub-layer of the roughness surfaces up to the free stream. The trend of the friction factor at the roughness bed region for the $10.0 \mathrm{~mm}$ case follow identical pattern compared to the $7.0 \mathrm{~mm}$ bed. The friction factor decreased progressively with increasing $\frac{a}{k_{s}}$ as shown in Figure 10b

The friction factor or wall friction as it may be referred is calculated from the maximum value of $u_{*}$ so that $f_{w}=2\left(\frac{u_{*}}{u_{m}}\right)^{2}$. The sudden drop in the friction factor at about $\frac{a}{k_{s}}=115$ as seen in Figure

10b marks the end of the transitional flow from the laminar into turbulence. A similar phenomenon occurred in the region of the boundary layer thickness at Reynolds numbers upwards of 1.0x $10^{4}$ as seen in Figure 11b when the development of boundary layer depth, $\delta$, started to occur in the free stream. The boundary layer depth, $\delta$, reduces with increasing flow described in the amplitude Reynolds number.

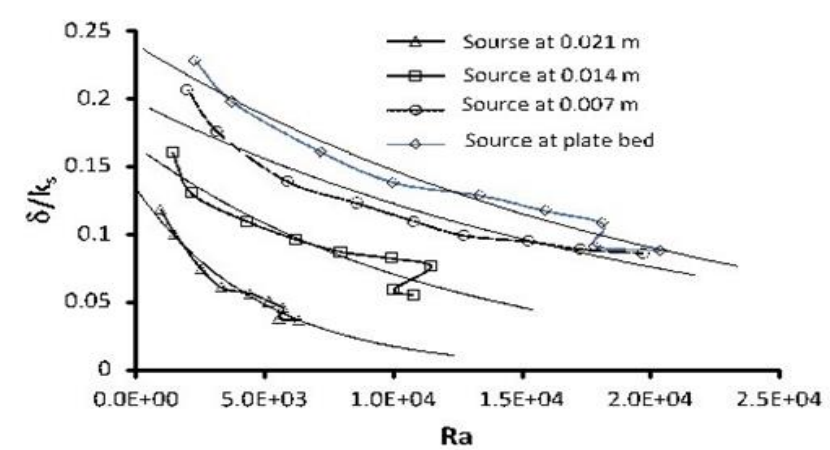

(a)

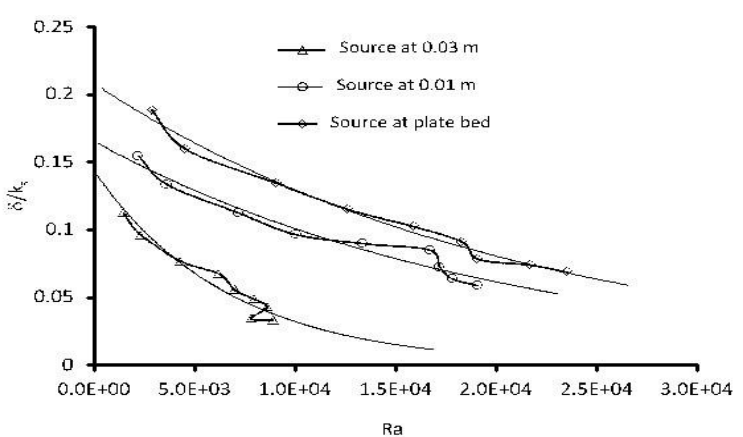

(b)

Figure 11: BL Thickness Response with Ra Flow at Varied Source Levels Below Porous Surface for the $7 \mathrm{~mm}$ and $10 \mathrm{~mm}$ Cases Respectively

\section{Conclusion}

Two types of porous bed setting with synthetic spherical beads $(7 \mathrm{~mm}$ and $10 \mathrm{~mm})$ were investigated in a flume bath. The dye tracer plume just above the roughness boundary layer and 
the cavity region (interfacial and subsurface sub-layers) of the flat plate attached to the oscillatory mechanism in the flume bath was monitored during the experiments. During the oscillatory flow, the crest of roughness elements induce elevation zones to force (advection) the flow upwards and pull it down at the trough phases as the tracer plume scales over the obstacles, establishing low and high pressure zones in the flow. The enhanced particles in the process mix and dissolve or decay faster with increasing oscillatory frequency, resulting into inter-particle collisions which initiate the conditions of advection and shear dispersion. Several authors (Smart, 1999; Nezu and Nakagawa, 1993; Choi and Waller, 1997; Prinos et al, 2003) have shown their findings with regard to the build-up of pressure relative to the behavior of the tracer plume as a consequence of the oscillatory mechanism. However, in the $10 \mathrm{~mm}$ preform the velocities relatively reduced inside the roughness which may be attributed to the larger pore size of the troughs and lower roughness height. In a study Prinos et al (2003) affirmed that the fluid's kinetic energy penetrating the porous medium increases with the Darcy number which is dependent on the structural geometry of the roughness elements (the pore size of the cavities or gaps between the elements).

The study viewed the phenomenon as a combination or coupling of the processes of gradient fluctuation enhanced through dispersion due to pressure forcing in and out of the macroscopic pore-fluid field caused by the oscillatory motions. The lightening as the dye-particles cascade in the porous medium can be associated to mixing-gain due to the enhanced oscillatory motion. Hydraulic conductivity was considered to increase with increasing pore-size and rates of tracer particle interactions within the cavities (Itugha, 2008).

The difference in the porosity between the $10 \mathrm{~mm}$ and $7 \mathrm{~mm}$ beds changes the velocity distribution and the turbulent characteristics of the flow. For instance, the velocities over the $10 \mathrm{~mm}$ bed had greater penetration of flow, hence were relatively higher in comparison with the flow over the $7 \mathrm{~mm}$ preform. The velocities of the different porous beds increased due to the high turbulence inside the roughness trough in comparison to the roughness height region (which is the distance from the roughness tops to roughness trough). Hydraulic conductivity was considered to increase with rates of tracer particle interactions within the cavities at the $10 \mathrm{~mm}$ pore-size.

\section{References}

[1] An, Y.Q. and Chen, D. (2004), A PDA study of sediment transport in a sediment-laden flow for the estimation of pollutant fluxes at water/sediment interface, 4th International Symposium on Environmental Hydraulics, Hong Kong.

[2] Choi, C. Y., and Waller, P. M. (1997). Momentum transport mechanism for water flow over porous media, Journal of Environmental Engineering, Vol. 123, No. 8, pp. 792-799

[3] Davis, A. M. J., and James, D. F. (2003), The Slip Velocity at the Edge of a Porous Medium: Effects of Interior Resistance and Interface Curvature. Transport in Porous Media, Vol. 53, pp. 175-196

[4] Einstein, Albert (1905). "Über die von der molekularkinetischenTheorie der WärmegeforderteBewegung von in ruhendenFlüssigkeitensuspendiertenTeilchen" (English translation: "Investigations on the theory of Brownian movement"). Annalen der Physik. 17 (8): 549-560. doi:10.1002/andp.19053220806.

[5] Engelund, F. (1970). Instability of erodible beds. Journal of Fluid Mechanics, 42 (1970), pp. 225244.

[6] Fredsøe, J. (1984), Turbulent boundary Layer in Wave-Current Motion. ASCE J. Hydraulic Engineering, vol. 110, No. 8, pp.1103-1120. 
[7] Huettel, M., Ziebis, W., Forster, S., and Luther III, G. W. (1998), Advective Transport affecting Metal and Nutrient Distributions and Interfacial Fluxes in Permeable Sediments, GeochimicaetCosmochimicaActa, Vol. 62, No. 4, pp. 613-631.

[8] Itugha, O.D., (2008). Solute and Particulate Transport at the Interface of Near-shore Permeable Estuarine Beach-sand: an Experimental Study of the Outer Reaches of River Mersey Estuary, N W England. Harold Cohen. THESIS 20860.ITU, Liverpool: Thesis Ph.D., 2008.

[9] Jensen, B. L., Sumer, B. M. and Fredsoe, J. (1989). Turbulent Oscillatory Boundary Layers at Reynolds Numbers, J. Fluid Mechanics, Vol. 206, pp. 265-297

[10] Keramaris, E. (2015). Effects of inclined impermeable bed on the turbulent characteristics of the flow using particle image velocimetry. Journal of Turbulence, 16 (6), 540-554.

[11] Keramaris, Evangelos (2017). Turbulent structure in uniform inclined open channel flow over different rough porous beds. International Journal of Sediment Research 32 (2017) 45-52.

[12] Krstic, R.V. \& Fernando, H.J.S. (2001). The nature of rough-wall oscillatory boundary layers Journal of Hydraulic Research, Volume 39, Issue 6, Pages 655-666.

[13] Nezu, I., and Nakagawa, H. (1993). Turbulence in open channel flow. IAHR Monograph, Balkema, Rotterdam, the Netherlands.

[14] Nielsen, P., Robert, S., Moller-Christiansen, B., Oliva, P. (2001). Infiltration effects on sediment mobility under waves. Coastal Engrg., 42, pp. 105-114.

[15] Nikora, V., S. McLean, S. Coleman, D. Pokrajac, I. McEwan, L. Campbell, J. Aberle, D. Clunie and K. Koll (2007b). Double-Averaging concept for rough-bed open-channel and overland flows: Applications. Journal of Hydraulic Engineering, 133(8): 884-895.

[16] Obhrai C., Nielsen P., Vincent C.E. (2002). Influence of infiltration on suspended sediment under waves. Coastal Engineering 45: pp111- 123.

[17] Pechlivanidis, G., Keramaris, E.,\&Pechlivanidis,I.(2012).Measurementsofturbulent characteristics in an open channel using PIV (particle image velocimetry). Global Nest Journal, 14(3), 378-385.

[18] Prinos, P., Sofialidis, D., and Keramaris, E. (2003). Turbulent flow over and within a porous bed. J. Hydraulic Engrg. Vol. 129 No. 9, pp. 720-733.

[19] Raupach, M.R., Antonia, R.A., Rajagopalan, S. (1991). Rough-wall turbulent boundary layers. Appl. Mech. Rev. Vol. 44, pp. 1-25.

[20] Shams, M, Currie, I. G., James, D. F. (2003). The flow field near the edge of a model porous medium. Experiments in Fluids, Vol. 35, pp. 193-198.

[21] Shim, Jaeho and Duan, Jennifer G. (2017). Experimental study of bed-load transport using particle motion tracking. International Journal of Sediment Research 32 (2017) 73-81.

[22] Smart, G. M. (1999). Turbulent velocity profiles and boundary shear in gravel bed rivers. J. Hydraulic Engrg. Vol. 125, No. 2, pp. 106-116.

[23] Stansby, P. K. (2003). A mixing-length model for shallow turbulent wakes. J. Fluid Mech., Cambridge, UK., Vol. 495, pp. 369-384.

[24] Suga, K. and Kuwata, Y. (2014). Turbulence over/inside porous surfaces and challenges to its modelling Journal of Physics: Conference Series 530 (2014) doi:10.1088/1742$6596 / 530 / 1 / 012004$.

[25] Suga, Kazuhiko (2016). Understanding and Modelling Turbulence Over and Inside Porous Media. Flow, Turbulence and Combustion. April 2016, Volume 96, Issue 3, pp 717-756. DOI 10.1007/s10494-015-9673-6.

[26] Svensson, U., and Rahm, L. (1991). Towards a mathematical model of oxygen transfer to and within bottom sediments. Journal of Geophysics Research, Atmos., Vol. 96, pp. 2777-2783.

*Corresponding author.

E-mail address: itughaod@ fuotuoke.edu.ng/emadigio@ yahoo.com 